\title{
Wer und wie wird gefördert? Eine kritische Analyse der KHG-Investitionsdaten
}

\author{
Boris Augurzky und Adam Pilny \\ (c) Der/die Autor(en) 2019 \\ J. Klauber et al. (Hrsg.), Krankenhaus-Report 2019 \\ https://doi.org/10.1007/978-3-662-58225-1_14
}

\section{Zusammenfassung}

Die Finanzierung der Investitionskosten im deutschen Krankenhauswesen ist seit Jahren Gegenstand politischer Debatten. Nicht zuletzt aufgrund der im Zeitverlauf sinkenden Fördermittel der Länder rückt dieses Thema immer weiter in den Vordergrund. Als Datengrundlage zur Messung des Fördermittelvolumens nach KHG dient für diese Diskussion die Statistik der Arbeitsgemeinschaft der Obersten Landesgesundheitsbehörden (AOLG). Dieser Beitrag zeigt, dass es Abweichungen im Fördervolumen zwischen der AOLG-Statistik und anderen Quellen zur Investitionsförderung gibt. Somit stellt sich die Frage, ob die AOLG-Statistik das tatsächliche Fördervolumen korrekt abbildet. Für die Jahre 2010 bis 2016 wird die AOLG-Statistik (i) den Eigenangaben der Landesministerien für Gesundheit aus einer Befragung sowie (ii) den Soll- und Ist-Ausgaben zur Investitionsförderung aus den Landeshaushalten gegenübergestellt. Darüber hinaus wird gezeigt, wie die Länder die Fördermittel unter den Krankenhausträgern aufteilen und welche Rolle Sonderfördermaßnahmen bei der Investitionsfinanzierung der Krankenhäuser spielen.

The financing of capital costs of German hospitals has been subject of political debate for many years. This issue is gaining in importance since the German federal states do not fulfill their legal obligation to fund the capital costs of hospitals sufficiently. The main data source for the states' capital funding is the statistic of the consortium of the supreme health care authorities of the German federal states (AOLG). This article contributes to the current debate in two ways: First, it reveals deviations between the AOLG statistic and other data sources on public capital funding for hospitals. This article compares the AOLG statistic for the period 2010 to 2016 with (i) funding data of the ministries of health of the German states obtained from a survey, and (ii) target and actual expenditures for investment promotion from the German states' budget. Second, this article provides analyses on capital funding by hospital ownership types and it sheds light on the role of special capital funding ("Sonderförderung") in addition to regular capital funding according to the hospital financing act (KHG).

\subsection{Hintergrund}

Die Finanzierung der Investitionskosten im deutschen Krankenhauswesen steht in gesundheitspolitischen Debatten nach wie vor weit oben auf der Agenda. Von zahlreichen Akteuren wird das er- brachte Investitionsvolumen der Länder als unzureichend empfunden. Als Nachweis, dass die Länder ihrer gesetzlichen Verpflichtung nicht in ausreichendem Maße nachkommen und sich zunehmend aus der Investitionsfinanzierung zurückziehen, wird die Statistik der Arbeitsgemeinschaft der 


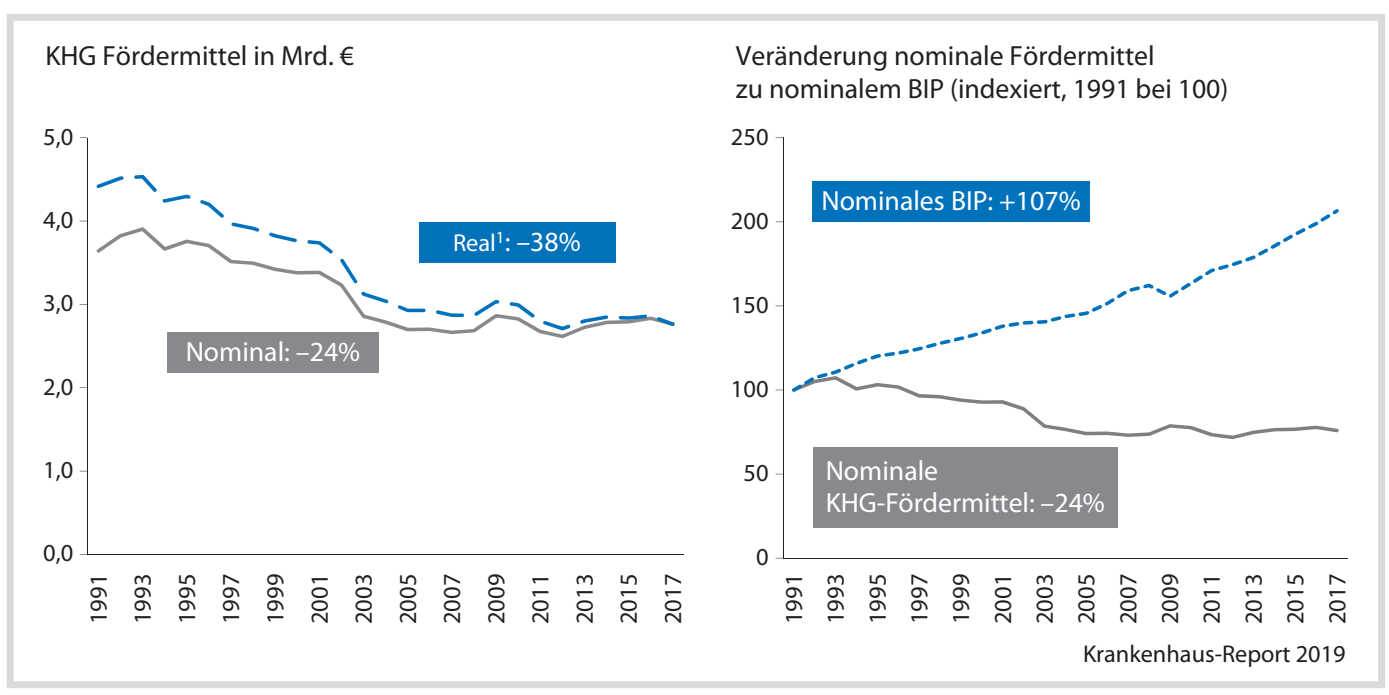

- Abb. 14.1 KHG-Fördermittel nach AOLG.

1 Deflationiert mit Investitionsgüterpreisindex

(Daten aus: Augurzky et al. 2018; DKG 2018; Statistisches Bundesamt 2018a, 2018b)

Obersten Landesgesundheitsbehörden (AOLG) herangezogen. - Abb. 14.1 zeigt die KHG-Ausgaben nach AOLG für den Zeitraum 1991 bis 2017. Nominal sind die KHG-Ausgaben demnach um 24 Prozent zurückgegangen, real sogar um 38 Prozent. Indessen stieg im gleichen Zeitraum das Bruttoinlandsprodukt um 107 Prozent an.

Im Rahmen der Erstellung des Gutachtens „Stand und Weiterentwicklung der Investitionsförderung im Krankenhausbereich" (RWI 2017), das das RWI - Leibniz-Institut für Wirtschaftsforschung e. V. im Auftrag des Bundesministeriums für Gesundheit erstellt hat, wurde eine Umfrage unter den Landesministerien für Gesundheit durchgeführt. In dieser Umfrage wurden die Landesministerien unter anderem zu ihren Investitionsausgaben nach KHG befragt. Die Länderangaben zu den KHG-Ausgaben wichen zum Teil um über 40 Prozent nach oben und unten von den Zahlen in der AOLG-Statistik ab. Im RWI-Gutachten konnte diese Diskrepanz zwischen beiden Quellen nicht abschließend geklärt werden (RWI 2017). Es blieb lediglich die Vermutung, dass die AOLG-Statistik keine tatsächlichen KHG-Ausgaben, sondern die Soll-Zahlen zu diesen Ausgaben darstellt. In der Anmerkung zu der AOLG-Statistik steht, dass die Angaben auf den jeweils öffentlich zugänglichen jährlichen Haushaltsansätzen der Länder beruhen (DKG 2018). Jedoch müssen Haushaltsansätze (Soll) nicht zwangsläufig mit dem Haushaltsvollzug (Ist) übereinstimmen. Eine weitere Erklärung für die Abweichungen wurde in der Rolle von Sonderfördermaßnahmen jenseits des KHG gesehen. Ob und in welchem Umfang diese in der AOLG-Statistik einfließen, blieb im RWI-Gutachten offen (RWI 2017). An dieser Stelle setzt der vorliegende Artikel an und will Transparenz schaffen.

Dieser Artikel gliedert sich in zwei Teile. Im ersten Teil werden die Zahlen der AOLG-Statistik für die Jahre 2010 bis 2016 sowohl den Länderangaben aus der RWI-Befragung als auch den Soll- und IstZahlen aus den Landeshaushalten gegenübergestellt. Im zweiten Teil werden Detailanalysen zu den KHG-Ausgaben vorgestellt und diskutiert. Hierbei wird auf die Ist-Zahlen aus den Haushaltsrechnungen der Länder zurückgegriffen, da diese detaillierte Analysen erlauben. Neben der Förderung nach Trägern wird die Rolle von Sonderfördermaßnahmen bei der Investitionsfinanzierung kritisch unter die Lupe genommen. 


\subsection{Daten}

Für diesen Artikel werden verschiedene Datenquellen verwendet, die den Zeitraum 2010 bis 2016 abdecken. Im Folgenden werden die Quellen kurz beschrieben:

1. AOLG-Statistik: Diese Statistik wird in unregelmäßigen Abständen von der Deutschen Krankenhausgesellschaft (DKG) in ihrer „Bestandsaufnahme zur Krankenhausplanung und Investitionsfinanzierung in den Bundesländern“ veröffentlicht. In der aktuellen Fassung werden die KHG-Investitionsfördermittel je Land für die Jahre 1991 bis 2017 angegeben (DKG 2018). Als Quelle dieser Statistik wird stets die Umfrage der Arbeitsgruppe für Krankenhauswesen der AOLG genannt. ${ }^{1}$

2. Eigenangabe der Länder: Für die Studie „Stand und Weiterentwicklung der Investitionsförderung im Krankenhausbereich“" (RWI 2017) wurden zwischen November 2016 bis Januar 2017 die Landesministerien für Gesundheit durch das RWI befragt. Im Rahmen des Fragebogens wurden zum einen die KHGAusgaben für die Jahre 2010 bis 2016 erhoben. Darüber hinaus wurde auch abgefragt, welche Sonderfördermaßnahmen - über das KHG hinaus - die Länder zwischen 1991 bis 2016 geleistet haben. Bis auf Sachsen haben sich alle Länder an der Befragung beteiligt.

3. Haushaltspläne und -rechnungen der Länder: Aus diesen Veröffentlichungen werden die Soll- und Ist-Werte zur Investitionsförderung von Krankenhäusern aus den Haushaltsjahren 2010 bis 2016 entnommen. Bei den Ist-Ausgaben handelt es sich um die im abgelaufenen Haushaltsjahr tatsächlich geleisteten Ausgaben. Die Soll-Ausgaben entsprechen den veran-

1 In einigen DKG-Veröffentlichungen werden neben der Umfrage der AOLG auch "eigene Berechnungen" als Quelle angegeben (vgl. DKG 2007, DKG 2009 und DKG 2018), während dieser Zusatz in den übrigen Veröffentlichungen zu der „Bestandsaufnahme" fehlt. Da in den letzten „Bestandsaufnahmen" (bis auf DKG 2018) ausschließlich die AOLG als Quelle angegeben worden ist und für die nachfolgenden Analysen diese Veröffentlichungen für den Zeitraum 2010 bis 2016 zugrunde gelegt werden, wird im Folgenden lediglich von der "AOLG-Statistik" gesprochen. schlagten Ausgaben, die im Laufe des betrachteten Haushaltsjahrs voraussichtlich zu leisten sind. Für einige Länder liegen die Ist-Daten nicht durchgehend vor, da diese in den öffentlich zugänglichen Daten nicht oder nur zum Teil ausgewiesen sind. ${ }^{2}$ Die Soll-Daten liegen dagegen für alle Länder durchgehend vor.

Da sich Aufbau und Darstellung der Investitionsausgaben für Krankenhäuser in den Haushaltsplänen und -rechnungen zwischen den Bundesländern unterscheiden, wurde wie folgt vorgegangen, um eine konsistente und valide Datengrundlage sowie eine Vergleichbarkeit zur AOLG-Statistik zu gewährleisten:

- Aus den Haushaltsplänen und -rechnungen wurden jeweils die Kapitel „Krankenhausfinanzierung “ bzw. „Krankenhausförderung“ zugrunde gelegt. In einigen Ländern finden sich die KHG-Ausgaben stattdessen in der "Allgemeinen Finanzverwaltung" oder unter dem „Kommunalen Finanzausgleich“' ${ }^{3}$ In einigen Fällen sind Sonderförderprogramme für Krankenhäuser auch in anderen Kapiteln ausgewiesen worden.

- Aus den Haushaltsplänen wurden die Ist- und Soll-Ausgaben nach KHG entnommen. Zum Teil haben die Länder auch eine Investitionsförderung für Krankenhäuser ausgewiesen, die jenseits des KHG erfolgte (Sonderförderung).

- Aus den Haushaltsrechnungen wurden die Ist-Ausgaben nach KHG entnommen. Diese wurden mit den Ist-Ausgaben aus den Haushaltsplänen abgeglichen, um eine valide Datenbasis zu gewährleisten.

- Verwaltungsausgaben und sächliche Ausgaben (z. B. für Gutachter und Sachverständige), die zwar in den Landeshaushalten unter „Krankenhausfinanzierung“ bzw. „Krankenhausförderung" ausgewiesen werden, sind nicht

2 Für folgende Länder und Jahre liegen keine Ist-Daten zu den KHG-Ausgaben vor: Schleswig-Holstein (2010), Mecklenburg-Vorpommern $(2011,2013,2015)$ und Berlin (2011, 2013, 2015).

3 Die Bezeichnungen der jeweiligen Kapitel können sich zwischen den Ländern unterscheiden und auch andere Positionen enthalten (z. B. Gesundheit allgemein, Rettungsdienste etc.). 
berücksichtigt worden. Es wurden ausschließlich die Positionen aufsummiert, die eindeutig als Investitionen und Schuldendiensthilfe (Zinsen und Tilgung) für Krankenhäuser ausgewiesen waren.

- Die Verpflichtungsermächtigungen ${ }^{4}$ für die Folgejahre, die in den Landeshaushalten für KHG-Ausgaben sowie Sonderförderung vorgesehen sind, wurden nicht berücksichtigt.

- Strukturfondsmittel nach $\$ 12$ KHG wurden herausgerechnet (vgl. DKG 2018). Für das Jahr 2015 hat Niedersachsen für „Strukturverbesserungen im ländlichen Raum" rund 4,9

Mio. Euro ausgegeben. ${ }^{5}$ Im Jahr 2016 haben drei Länder Mittel aus dem Strukturfonds ausgezahlt: NRW mit 16,6 Mio. Euro, RheinlandPfalz mit 6,5 Mio. Euro und Mecklenburg-Vorpommern mit 2,5 Mio. Euro.

- Die in den Landeshaushalten ausgewiesenen Ausgaben zur Investitionsförderung von Krankenhäusern enthalten ferner keine Investitionsmittel von Hochschulkliniken, Bundeswehrkrankenhäusern und Vertragskrankenhäusern oder Eigenmittel der Plankrankenhäuser.

- Aus den Haushaltsplänen und -rechnungen wurden jeweils alle Daten erfasst, die zweifelsfrei als Investitionsausgaben bzw. -förderung für Krankenhäuser zugeordnet werden konnten. Das gilt neben den regulären KHG-Ausgaben auch für Mittel aus Sonderförderprogrammen, sofern diese nach den Zuwendungsempfängern differenziert aufgelistet waren. Sollte es in einigen Ländern Sonderförderungen für Krankenhausinvestitionen gegeben haben, die im Haushalt nicht in Einzelpositionen nach Zuwendungsempfängern, sondern nur als Sammelposition dargestellt sind, so stel-

4 Sind öffentlich geförderte Investitionen auf mehrere Jahre angelegt (z. B. Bau eines neuen Krankenhausgebäudes), ist es notwendig, dass die Länder bereits in einem früheren Haushaltsjahr Verpflichtungen zur Förderung von Investitionsmaßnahmen eingehen, die erst in späteren Haushaltsjahren zu Ausgaben führen. Hierzu werden Verpflichtungsermächtigungen im Haushaltsplan veranschlagt.

5 Die Ausgabe von Mitteln aus dem Strukturfonds ist eigentlich erst ab 2016 vorgesehen. In der niedersächsischen Haushaltsrechnung 2015 sind diese Ist-Ausgaben jedoch angegeben. len die in diesem Artikel abgebildeten Sollund Ist-Zahlen die untere Grenze der KHGAusgaben dar. ${ }^{6}$

\subsection{AOLG-Statistik im Vergleich zu anderen Datenquellen}

Für die Jahre 2012, 2014 und 2016 liegen für alle 16 Länder die Daten aus den Haushalten vollständig vor. - Abb. 14.2 zeigt die Differenz zwischen der AOLG-Statistik aus DKG (2018) und den Ist- und Soll-Zahlen aus den Landeshaushalten. Die Ist-Ausgaben zur Investitionsförderung lagen im Bundesdurchschnitt 2012 um 40 Mio. Euro (1,5\%) über den von der AOLG ausgewiesen KHG-Ausgaben, wohingegen sie 2014 um 188 Mio. Euro (-6,8 \%) und 2016 um 50 Mio. Euro (-1,7 \%) geringer ausfielen als in der AOLG-Statistik. Indessen weichen die Soll-Ausgaben kaum von der AOLG-Statistik ab (jeweils um $0 \%$ ). Für diese drei Jahre würde die AOLG-Statistik die tatsächlichen KHG-Ausgaben um rund 200 Mio. Euro bzw. 2,4 Prozent überschätzen. Im Bundesdurchschnitt und über die Zeit fallen die Differenzen zwischen der AOLGStatistik und den Ist-Ausgaben nicht schwer ins Gewicht. Bei einem Vergleich für den gesamten Zeitraum 2010 bis 2016 - basierend auf den Ländern mit verfügbaren Daten ${ }^{7}$ - würde die Abweichung mit -2,9 Prozent eine ähnliche Größenordnung aufweisen.

Auf Länderebene zeigt sich jedoch ein anderes, sehr heterogenes Bild, das deutlichere Abweichungen zeigt (• Abb. 14.3). Es fällt auf, dass es zwischen den AOLG- und Soll-Zahlen in Baden-Württem-

6 Beispielsweise könnten Fördermittel aus dem Zukunftsinvestitionsgesetz (Konjunkturpaket II) darunter fallen. Einige Länder haben explizit solche Mittel für Krankenhäuser ausgewiesen. Andere Länder haben hingegen solche Ausgaben als Sammelposition deklariert, sodass die Aufschlüsselung nach Zuwendungsempfängern unklar ist. Ob und inwieweit in diesen Fällen Krankenhäuser Fördermittel erhielten, bleibt an dieser Stelle offen. Für Details zum Zukunftsinvestitionsgesetz s. Abschn. 14.5.

7 Für die Jahre 2010, 2011, 2013 und 2015 liegen nicht für alle Länder Daten vor. Zur Berechnung der prozentualen Abweichung für den Zeitraum 2010 bis 2016 wurden diese Länder nicht berücksichtigt, sondern nur jene mit vorliegenden Daten. 

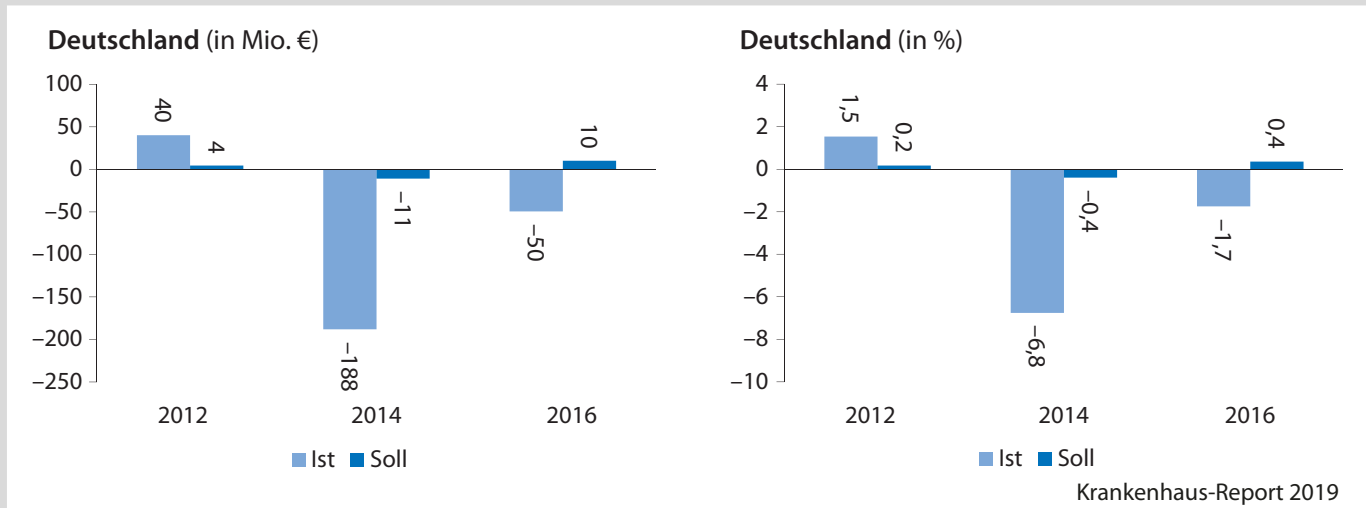

- Abb. 14.2 Abweichung zwischen AOLG-Statistik und Länder-Haushaltsdaten auf Bundesebene Anmerkung: Die AOLG-Statistik ist hier die Referenz. Bei negativen (positiven) Werten sind die KHG-Zahlen aus den Landeshaushalten geringer (höher) als in der AOLG-Statistik. Es werden nur die Jahre gezeigt, für die die Daten aller 16 Länder vollständig vorliegen. (Daten aus: DKG 2018; Haushaltspläne und -rechnungen der Länder, eigene Berechnung)

berg, Berlin, Hessen, Nordrhein-Westfalen und Rheinland-Pfalz für die Jahre 2010 bis 2016 keine Abweichungen gibt (schwarze Balken in der Abbildung). Auch in sechs weiteren Ländern gibt es bis auf ein oder zwei Jahre keine Differenz zwischen beiden Quellen. ${ }^{8}$ Dieses Ergebnis bestätigt, dass die AOLG-Statistik zum Großteil auf Soll-Zahlen anstelle von Ist-Zahlen beruht.

Allen Ländern gemein ist, dass es Abweichungen zwischen den Ist- und den AOLG-Zahlen gibt. In der Tendenz liegen die Ist-Werte unter den AOLG-Zahlen (hellgraue Balken in der Abbildung). Lediglich in Berlin und in Rheinland-Pfalz stimmen die Zahlen aller Quellen nahezu überein, was bedeutet, dass diese Länder Investitionsmittel-Ausgaben tatsächlich in der Höhe getätigt haben, die in den Haushaltsansätzen veranschlagt wurde. Die stärksten relativen Abweichungen gemessen am gesamten Fördervolumen in einzelnen Jahren weisen das Saarland (bis $+47 \%$ ), Mecklenburg-Vorpommern (bis $+44 \%$ ), Sachsen (bis $+35 \%$ und bis $-27 \%$ ), Hamburg (bis $+23 \%$ ) und Sachsen-Anhalt (bis $-17 \%$ ) auf. In neun Ländern gibt es über die Zeit Abweichungen in beide Richtungen. In Bayern,

8 Auch in Niedersachsen gäbe es eine vollständige Übereinstimmung zwischen Soll-Zahlen und den AOLG-Zahlen, würden die veranschlagten Mittel für "Strukturverbesserungen im ländlichen Raum" in Höhe von 4 Mio. Euro (2014) bzw. 3 Mio. Euro (2015) berücksichtigt werden.
Bremen, Nordrhein-Westfalen und Sachsen-Anhalt sind die tatsächlichen Ausgaben über den gesamten betrachteten Zeitraum geringer ausgefallen als in der AOLG-Statistik ausgewiesen. Hingegen fallen die Ist-Ausgaben lediglich in Thüringen höher aus als in der AOLG-Statistik.

Als weitere Quelle wurden die Eigenangaben der Länder herangezogen. In Baden-Württemberg, Bayern, Rheinland-Pfalz und im Saarland stimmen die Eigenangaben durchgehend mit der AOLGStatistik überein (blaue Balken in der Abbildung). In den anderen Ländern gibt es dagegen Abweichungen, die in Berlin, Niedersachsen und Thüringen am stärksten sind. Interessant ist die Übereinstimmung von Eigenangabe und Ist-Werten in Brandenburg, Hamburg (2014) und MecklenburgVorpommern (2016), sofern beide Werte von den AOLG-Zahlen abweichen.

\subsection{Investitionsförderung nach Trägerschaft}

Die Daten aus den Landeshaushalten von zwölf Ländern erlauben es, detaillierte Analysen zur Investitionsförderung von Krankenhäusern nach Trägern durchzuführen. Basierend auf den Ist-Ausgaben zeigt • Abb. 14.4 die Verteilung der Fördermittel nach Trägerschaft, wobei freigemeinnützige und private Träger zu „nicht-kommunal“ zusam- 


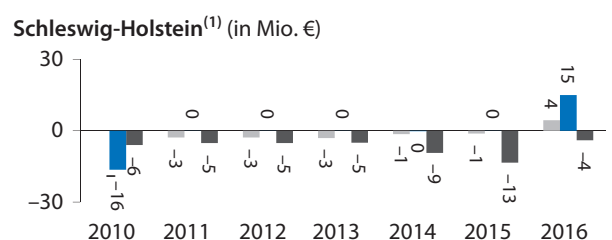

Hamburg (in Mio. €)

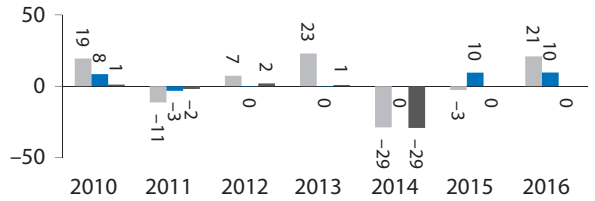

Niedersachsen (in Mio. €)

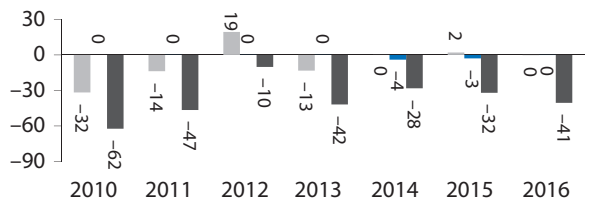

Bremen (in Mio. €)

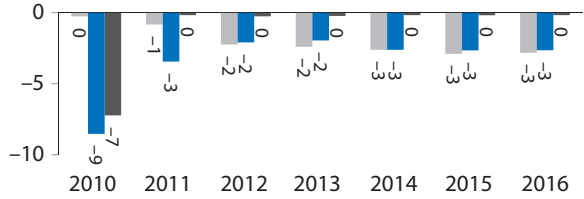

Nordrhein-Westfalen (in Mio. €)

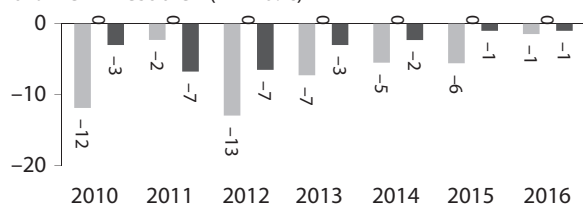

Hessen (in Mio. $€$ )

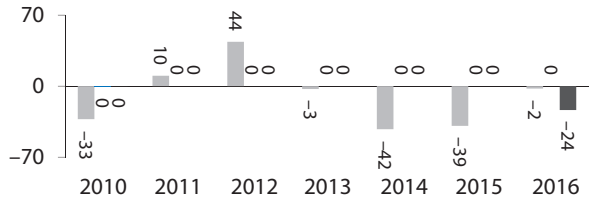

Ist
Schleswig-Holstein ${ }^{(1)}$ (in \%)

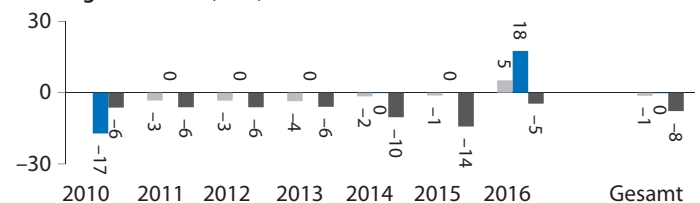

Hamburg (in \%)

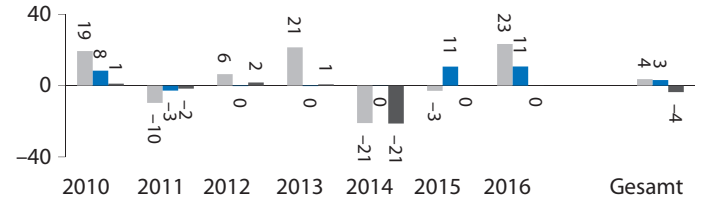

Niedersachsen (in \%)

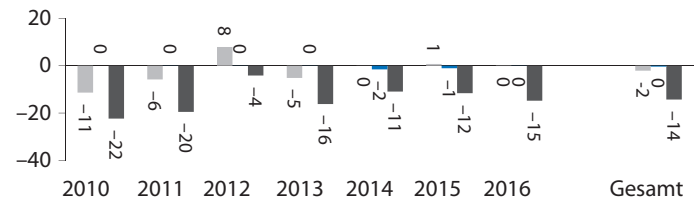

Bremen (in \%)

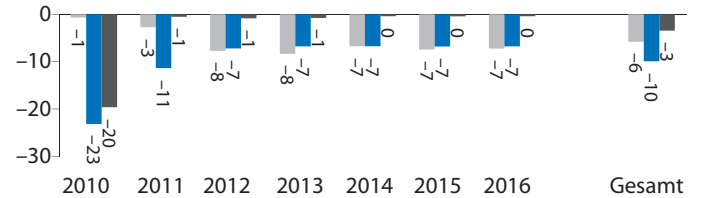

\section{Nordrhein-Westfalen (in \%)}

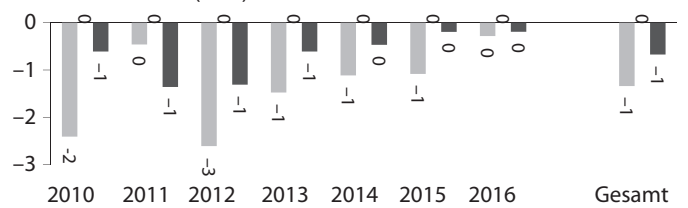

Hessen (in \%)

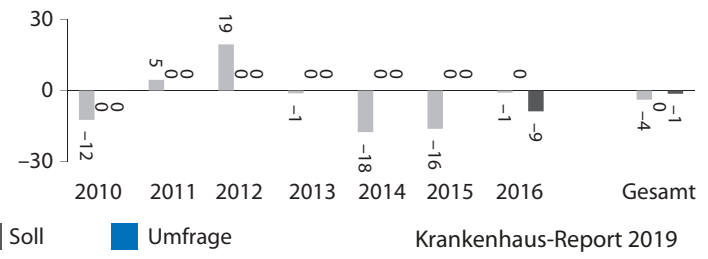

- Abb. 14.3 Abweichung zwischen AOLG-Statistik und Länder-Haushaltsdaten auf Länderebene in Mio. Euro und in Prozent (1) Ist-Daten für Schleswig-Holstein liegen für 2010 nicht vor.

(2) Ist-Daten liegen für Berlin 2011, 2013 und 2015 nicht vor.

(3) Ist-Daten liegen für Mecklenburg-Vorpommern 2011, 2013 und 2015 nicht vor.

(4) Für Sachsen liegen keine Eigenangaben vor, da Sachsen sich nicht an der RWI-Umfrage beteiligt hat.

Anmerkung: Die AOLG-Statistik ist hier die Referenz. Bei negativen (positiven) Werten sind die KHG-Zahlen aus den anderen Quellen geringer (höher) als in der AOLG-Statistik.

(Daten aus: DKG 2018; RWI 2017; Haushaltspläne und -rechnungen der Länder, eigene Berechnung) 


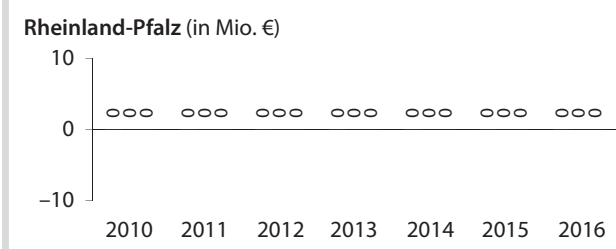

Baden-Württemberg (in Mio. €)

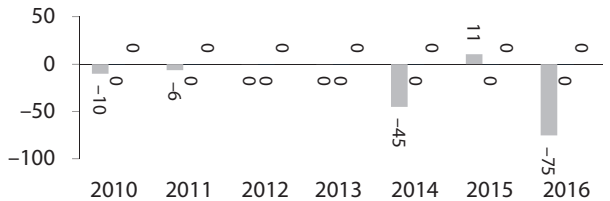

Bayern (in Mio. €)

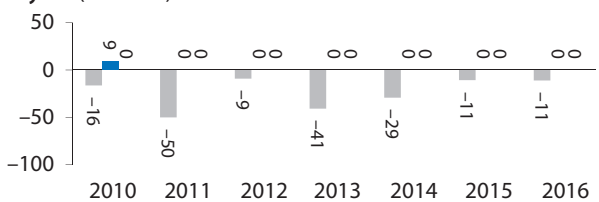

Saarland (in Mio. €)

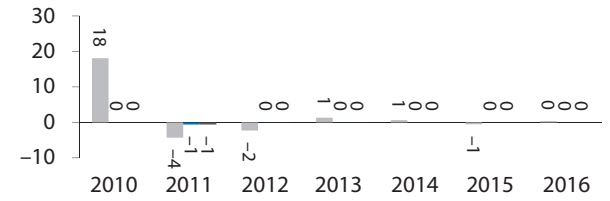

Berlin $^{(2)}$ (in Mio. €)

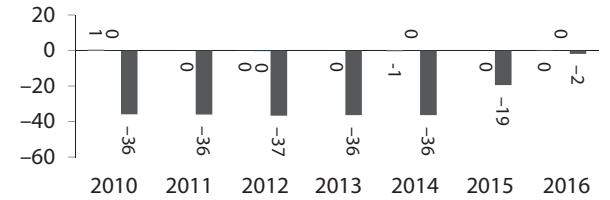

Brandenburg (in Mio. €)

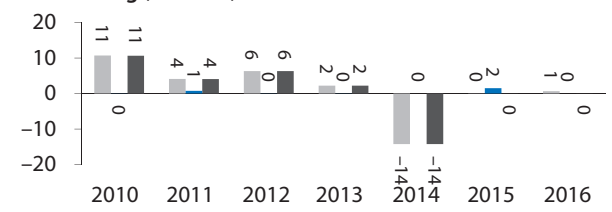

Rheinland-Pfalz (in \%)

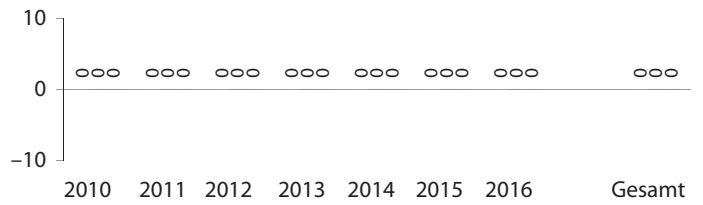

Baden-Württemberg (in \%)

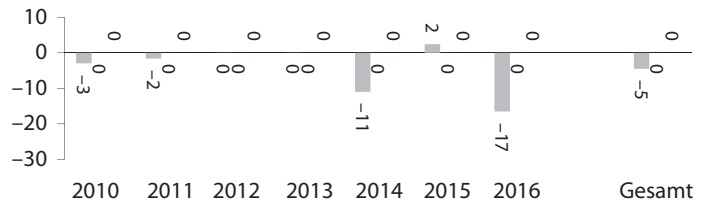

Bayern (in \%)

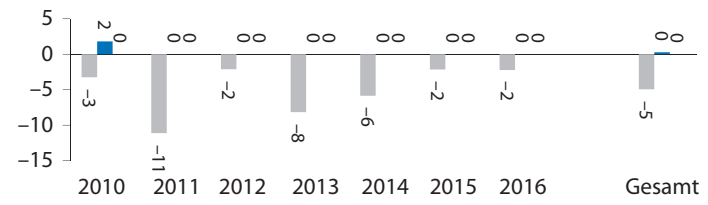

Saarland (in \%)

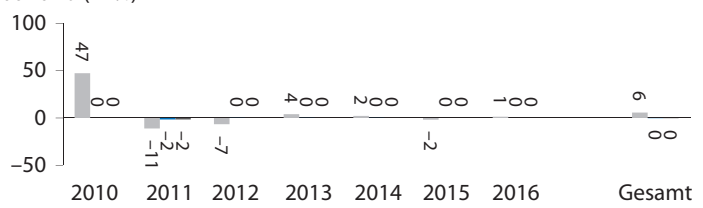

Berlin $^{(2)}$ (in \%)

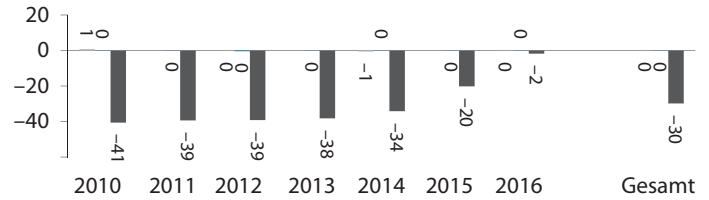

Brandenburg (in \%)

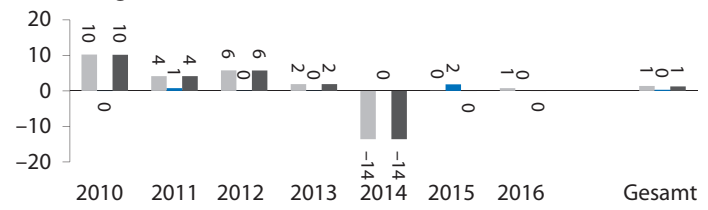

Soll Umfrage

Krankenhaus-Report 2019

- Abb. 14.3 (Fortsetzung) 


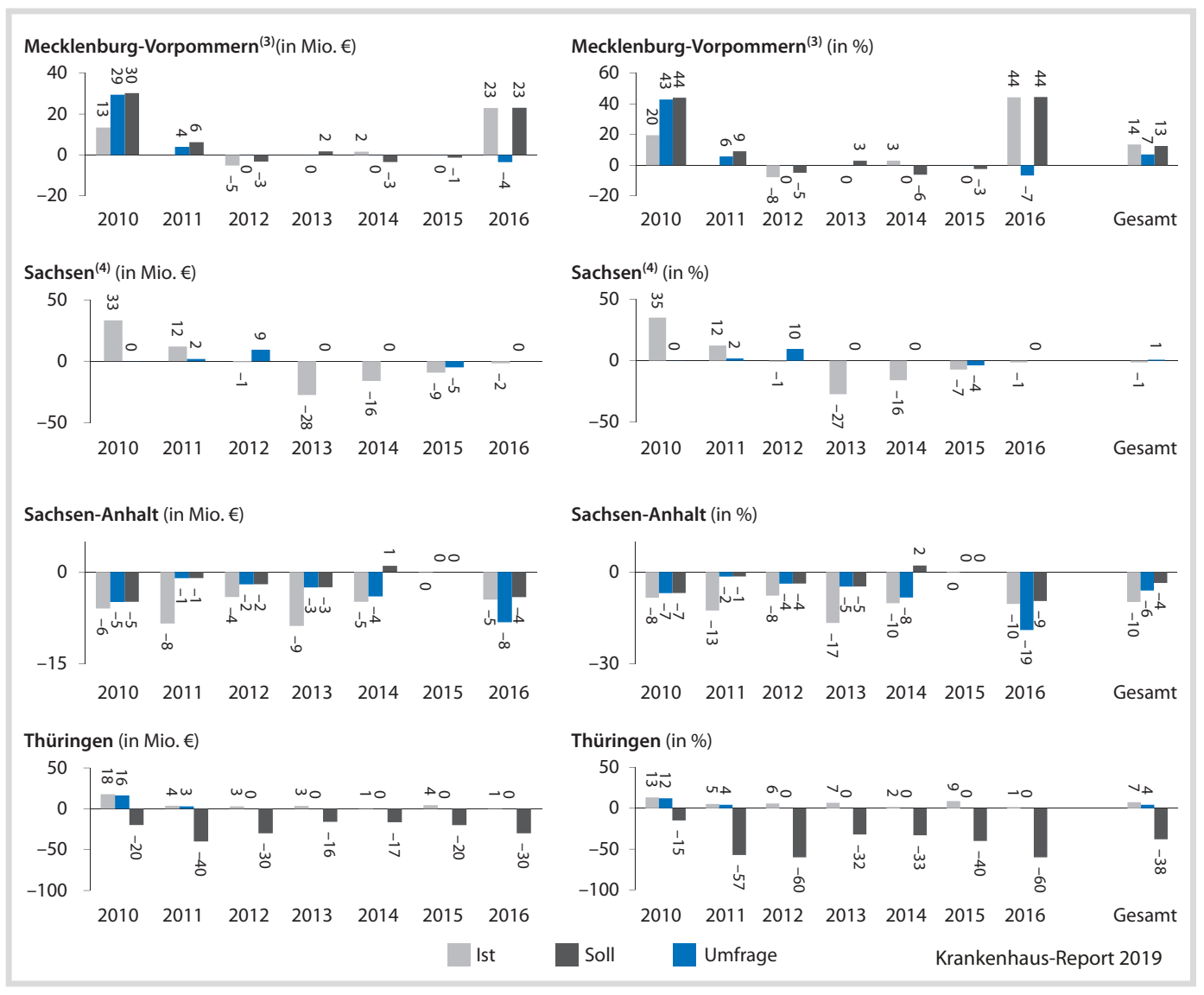

- Abb. 14.3 (Fortsetzung)

mengefasst werden. ${ }^{9}$ Auf den ersten Blick scheint es Länder zu geben, die ihre Fördermittel bevorzugt an kommunale Krankenhäuser vergeben, insbesondere Bremen, Bayern und Baden-Württemberg. Ein Großteil der Fördermittel geht hingegen in Mecklenburg-Vorpommern, Nordrhein-Westfalen und Rheinland-Pfalz an nicht-kommunale Träger.

Die Zusammensetzung der Krankenhausträger innerhalb der Länder könnte der wesentliche Grund dafür sein, dass ein Großteil des Fördervolumens überwiegend an Krankenhäuser mit einer bestimmten Trägerschaft verteilt wird. Für einen aussagekräftigeren Vergleich zeigt • Abb. 14.5 die Höhe der

9 Eine detailliertere Aufteilung der nicht-kommunalen Träger ist für den betrachteten Zeitraum nur für Niedersachsen möglich. Die anderen Länder fassen die KHG-Ausgaben für freigemeinnützige und private Träger stets zusammen.
Fördermittel für den Zeitraum 2010 bis 2016 je gefördertes Bett nach Trägerschaft und Ländern (s. linkes Diagramm) ${ }^{10}$ Kommunale Krankenhäuser erhielten demnach mit über 7.000 Euro je gefördertes Bett die höchste Förderung in BadenWürttemberg, Bayern, Berlin und Bremen. Am geringsten wurden kommunale Träger mit maximal 3.100 Euro je gefördertes Bett in Mecklenburg-Vorpommern und Nordrhein-Westfalen gefördert. In Mecklenburg-Vorpommern, Nordrhein-Westfalen und im Saarland erhielten nicht-kommunale Träger hingegen mehr Fördermittel je gefördertes Bett als kommunale Träger. In Mecklenburg-Vorpommern

10 Zur Berechnung der durchschnittlichen Fördermittel je Bett werden die nach KHG geförderten Betten aus den Grunddaten der Krankenhäuser des Statistischen Bundesamtes zugrunde gelegt. 


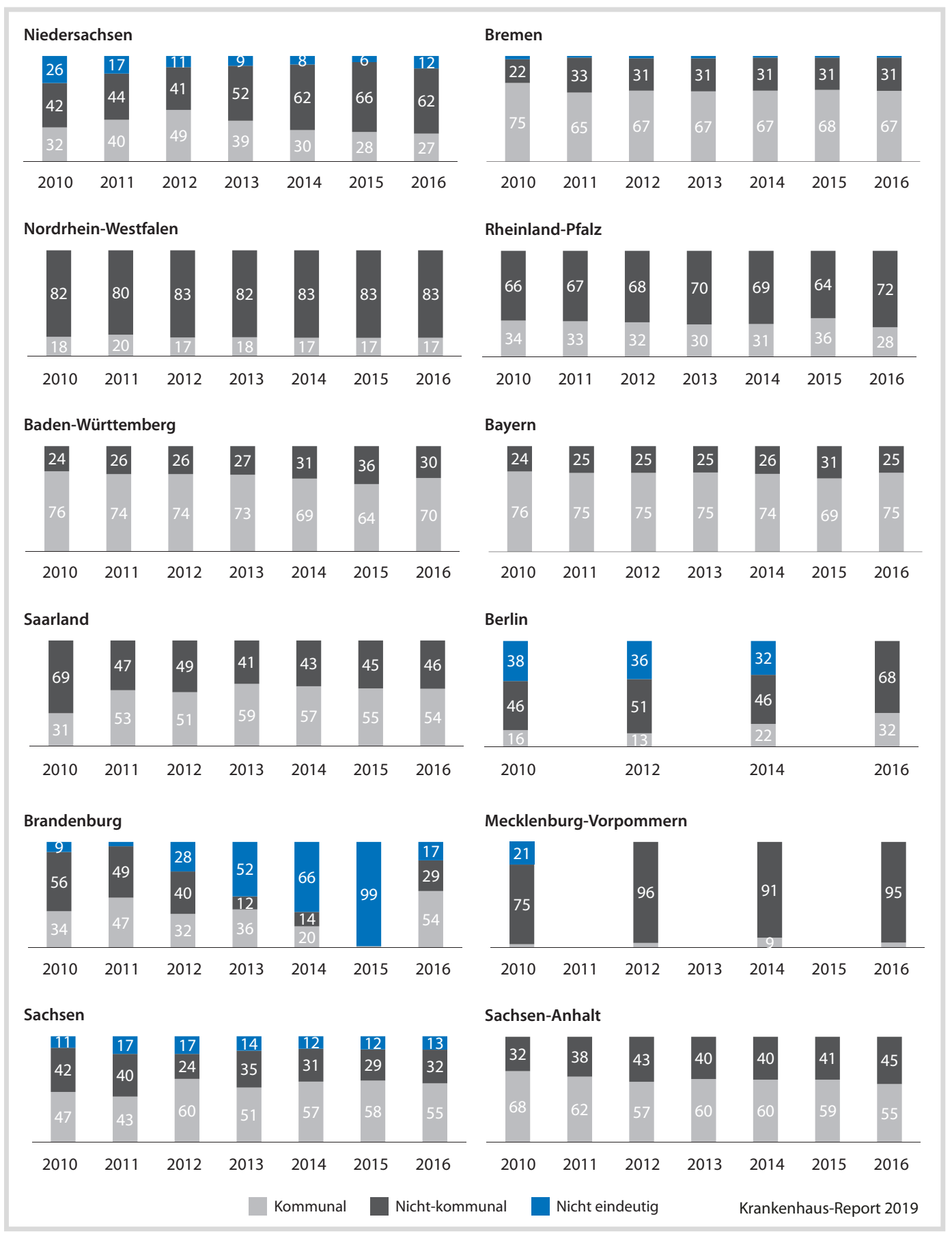

- Abb. 14.4 Verteilung der Fördermittel nach Trägerschaft in Prozent des gesamten Fördervolumens (Ist-Ausgaben) Anmerkung: Investitionsfördermittel, die keinen Trägern eindeutig zugeordnet werden konnten, sind unter der Kategorie „nicht eindeutig“ zusammengefasst.

(Daten aus: Haushaltspläne und -rechnungen der Länder, eigene Berechnung) 
wurden diese sogar mit über 9.000 Euro je Bett am stärksten gefördert, in Sachsen-Anhalt dagegen nur mit 2.600 Euro je gefördertes Bett. Die beiden weiteren Diagramme in - Abb. 14.5 zeigen, dass nicht alle Betten in den jeweiligen Bundesländern nach KHG gefördert wurden. ${ }^{11}$ Bei den Gesamtausgaben ist Mecklenburg-Vorpommern mit 6.300 Euro je Bett nicht mehr der Spitzenreiter (8.100 Euro je gefördertes Bett), was daran liegt, dass im Durchschnitt nur 78 Prozent aller Betten nach KHG gefördert wurden. Differenziert nach Trägerschaft zeigt sich, dass der Anteil geförderter Betten bei nicht-kommunalen Trägern in nahezu allen Ländern höher war als bei kommunalen Trägern. Gleichwohl gibt es in der Bandbreite dieser Förderquoten Unterschiede zwischen den Ländern. Es scheint jedoch, als hätten die Länder tendenziell nicht-kommunale Träger eher nach dem Gießkannenprinzip gefördert, während kommunale Träger vielmehr fokussiert gefördert wurden.

\subsection{Die Rolle von Sonder- förderungen}

Im RWI-Gutachten stellt sich die Frage, welche Rolle Sonderförderungen bei der Investitionsfinanzierung von Krankenhäusern spielen (RWI 2017). Hierzu wurden die Ministerien nach allen Sonderförderprogrammen seit 1991 befragt. Mehrere Länder haben in der RWI-Umfrage Sonderförderprogramme ab 2011 angegeben. Ausschließlich Thüringen hat für einen früheren Zeitraum (1994 bis 2001) Sonderinvestitionen angegeben. Mehrheitlich handelt es sich bei den angegebenen Sonderförderprogrammen um aktuelle Programme, deren Mittel erst in den nächsten Jahren ausgezahlt werden. ${ }^{12}$ In den Landeshaushalten wurden zum Teil neben diesen angegebenen Sonderförderungen auch solche Programme ausgewiesen, die die Länder nicht in der RWI-Umfrage angegeben haben. Im Einzelnen sind folgende Sonderförderprogramme zwischen 2010 bis 2016 für die Investitionsförderung in den Landeshaushalten angesetzt worden,

11 Hierbei werden die Betten aller Krankenhäuser in den Ländern zugrunde gelegt.

12 Für Details siehe Seiten 25 f. in RWI 2017. für welche die folgenden Ist-Ausgaben in den betroffenen Haushaltsjahren geleistet wurden:

- Im Rahmen des Konjunkturpakets II hat der Bundestag 2009 das sogenannte Zukunftsinvestitionsgesetz beschlossen, um Investitionen in Ländern und Gemeinden zu fördern. Ein Förderbereich war u. a. der Investitionsschwerpunkt Infrastruktur, der Investitionsförderungen für Krankenhäuser ermöglichte. ${ }^{13}$ Mehrere Länder haben 2010 und 2011 Investitionsfördermittel aus den Mitteln des Zukunftsinvestitionsgesetzes für Krankenhäuser ausgezahlt: Bremen (6,3 Mio. Euro), Bayern (36,6 Mio. Euro), Brandenburg (14,9 Mio. Euro), Mecklenburg-Vorpommern (17,6 Mio. Euro) $)^{14}$, Sachsen (19,4 Mio. Euro) und Thüringen (17,8 Mio. Euro). ${ }^{15}$

- Schleswig-Holstein: Besondere Landeszuschüsse für Investitionen an Krankenhausträger in Höhe von 16,4 Mio. Euro für 2015 und 2016.

- Nordrhein-Westfalen: Sonderfonds Krankenhäuser in Höhe von 550.000 Euro für 2014.

- Berlin: Kommunalinvestitionsprogramm in Höhe von 30 Mio. Euro für 2016.

- Sachsen: Maßnahmen im Gesundheits- und Sozialbereich aus Mitteln des Europäischen Fonds für regionale Entwicklung in Höhe von 8,7 Mio. Euro für 2012 bis 2014.

In den vorangehenden Analysen haben wir diese Summen mit den regulären KHG-Ausgaben zusammengelegt. Es zeigte sich, dass diese Mittel auch zum Teil in der AOLG-Statistik mitberücksichtigt wurden, da in den meisten Fällen die Soll-Zahlen inklusive der Sonderfördermittel mit den AOLGZahlen übereinstimmen. Andere Sonderförde-

13 Vgl. § 3 Abs. 1 Satz 2a ZulnvG. Ferner wurden auch Investitionen in den Bereichen Bildung, Städtebau, Straßenbau, Informationstechnologie etc. ermöglicht.

14 Für Mecklenburg-Vorpommern liegen keine Ist-Angaben für 2011 vor, sodass diese Sonderförderung prinzipiell auch höher ausgefallen sein könnte.

15 Andere Länder könnten ebenfalls Investitionsfördermittel aus diesem Programm für Krankenhäuser bereitgestellt haben, diese aber (i) nicht eindeutig im Landeshaushalt als solche ausgewiesen haben oder (ii) zu den KHGAusgaben hinzugezählt haben. Daher können an dieser Stelle keine Angaben zu den anderen Ländern gemacht werden. 


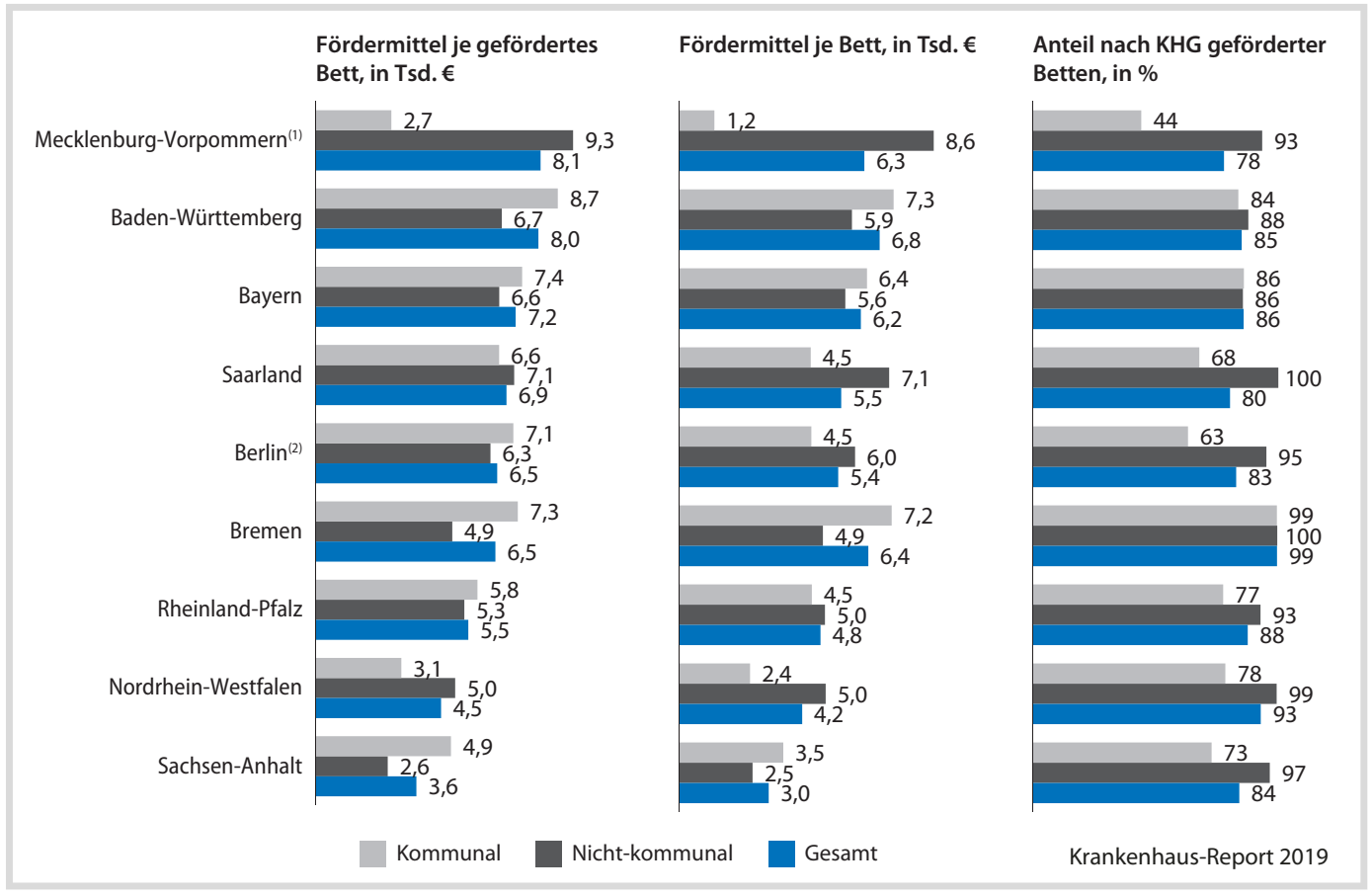

- Abb. 14.5 Fördermittel je gefördertes Bett nach Trägerschaft 2010 bis 2016, in Preisen von 2016

(1) Werte für Mecklenburg-Vorpommern beziehen sich nur auf den Durchschnitt der Jahre 2012, 2014 und 2016.

(2) Werte für Berlin beziehen sich nur auf 2016, da die Daten der anderen Jahre nicht eindeutig nach Trägerschaften differenzierbar sind.

Anmerkung: Im ersten Diagramm werden ausschließlich nur nach KHG geförderte Betten aus den Grunddaten der Krankenhäuser des Statistischen Bundesamtes zugrunde gelegt, während es im zweiten Diagramm die Betten aller Krankenhäuser sind. Brandenburg, Niedersachsen und Sachsen werden hier nicht gezeigt, da die Werte für 2010 bis 2016 durch die Kategorie „nicht eindeutig“ verunreinigt sind. In Bremen beträgt der Anteil der nicht-eindeutigen Fördermittel lediglich 1,9 Prozent, sodass hier eine mögliche Verunreinigung relativ gering ausfällt und die ausgezahlten Fördermittel je Bett leicht höher ausfallen. Die Werte sind mit dem Investitionspreisgüterindex deflationiert und in Preisen von 2016 dargestellt.

(Daten aus: Haushaltspläne und -rechnungen der Länder; Statistisches Bundesamt 2011, 2013a, 2013b, 2014, 2015, 2016, 2017, 2018a, eigene Berechnung)

rungsprogramme wie den Strukturfonds nach KHSG rechnet die AOLG-Statistik hingegen heraus (vgl. DKG 2018).

Bei der Sonderförderung von Krankenhausinvestitionen stellt sich die Frage, inwieweit die Länder Sonderförderprogramme als zusätzliche Förderung oder als Substitut der regulären KHGFördermittel einsetzen. Aus Sicht der Länder könnte es attraktiv sein, regulär zu leistende KHG-Ausgaben mit Mitteln aus anderen Töpfen zu substituieren. Die Aufstellung der Sonderförderprogramme oben zeigt, dass Mittel aus anderen Töpfen außerhalb der Landeshaushalte regelmäßig genutzt wurden. Diese Mittel sind zudem häufig mit einer
Kofinanzierung verbunden, wie beispielsweise durch den Bund im Rahmen des Zukunftsinvestitionsgesetzes (Konjunkturpaket II) oder des Kommunalinvestitionsprogramms. So hat Berlin im Jahr 2016 aus dem Kommunalinvestitionsprogramm vorgesehen zur Förderung strukturschwacher Regionen - 30 Mio. Euro für Krankenhausinvestitionen ausgegeben. 90 Prozent dieses Betrages wurden vom Bund kofinanziert. Bei einer Gesamtfördersumme von über 108,7 Mio. Euro hat sich Berlin im Jahr 2016 demnach 25 Prozent aller Ausgaben zur Investitionsförderung für Krankenhäuser vom Bund finanzieren lassen. Interessant ist dabei die Tatsache, dass 108,9 Mio. Euro im Haushaltsplan 
2016/2017 für die Investitionsförderung von Krankenhäusern im Jahr 2016 veranschlagt waren. Die Soll-Ausgaben wurden demnach auch tatsächlich getätigt, jedoch wurden bei den Ist-Ausgaben für Investitionspauschalen veranschlagte 30 Mio. Euro gekürzt und mit Mitteln aus dem Sonderprogramm substituiert.

\subsection{Fazit}

Die Investitionsfinanzierung von Krankenhäusern ist ein „Evergreen“ in gesundheitspolitischen Debatten. Insbesondere das unzureichende Investitionsvolumen der Länder wird von vielen Akteuren häufig beklagt. Umso wichtiger ist eine valide Datengrundlage, mit der sich die jährlich geleistete Investitionsförderung der Länder beziffern lässt. Bisher wurde in der Regel auf die Statistik der AOLG zurückgegriffen. Dieser Artikel zeigt, dass die AOLG-Statistik zum Großteil auf den Soll-Zahlen (Haushaltsansätzen) zu den KHG-Ausgaben beruht und nicht das tatsächlich geleistete Volumen der Investitionsförderung widerspiegelt. Im Bundesdurchschnitt weichen die Ist-Zahlen für die Jahre 2012, 2014 und 2016 nur geringfügig um -2,4 Prozent von der AOLG-Statistik ab. Auf Länderebene zeigt sich jedoch ein deutlich heterogeneres Bild mit Abweichungen zwischen -27 und +47 Prozent.

Ferner zeigt sich, dass die Länder die Krankenhausträger unterschiedlich stark fördern. Während in Süddeutschland sowie in Berlin und Bremen die kommunalen Träger im Zeitraum 2010 bis 2016 mit über 7.000 Euro je gefördertes Bett am stärksten gefördert wurden, fiel ihre Förderung in Mecklenburg-Vorpommern und Nordrhein-Westfalen mit maximal 3.000 Euro je gefördertes Bett deutlich geringer aus. Nicht-kommunale Krankenhäuser wurden indessen in Mecklenburg-Vorpommern mit über 9.000 Euro je Bett gefördert, in SachsenAnhalt dagegen nur mit 2.500 Euro je Bett. Schließlich wurde gezeigt, dass Sonderförderprogramme von den Ländern häufig zur Förderung von Krankenhausinvestitionen genutzt werden. Diese Programme gehen regelmäßig mit einer Kofinanzierung (z. B. durch den Bund) einher, sodass die Landesaufgabe der Krankenhausfinanzierung zu einem gewissen Umfang von anderen Geldgebern querfinanziert wird. Obwohl der Bund in den vergangenen Jahren teils in erheblichem Umfang Krankenhausinvestitionen mitfinanziert hat, hat er dafür keine adäquaten Mitspracherechte zur Gestaltung einer effektiven und effizienten Krankenhausversorgung erhalten.

Im Sinne einer angemessenen Beurteilung der tatsächlichen Investitionsförderung durch die Länder fordern wir eine valide und transparente Datengrundlage, die anstelle von Soll-Zahlen auf IstZahlen beruht. Für alle Akteure im Krankenhausmarkt ist eine solche Datenbasis essenziell, da sie die Grundlage von politischen Verhandlungsprozessen, insbesondere um zukünftige Investitionsmittel, darstellt. Verzerrte Zahlen zum KHG-Fördervolumen können und sollten nicht Grundlage solcher wichtigen Entscheidungen sein. Darüber hinaus sollte künftig klar abgrenzbar sein, welche Leistungen die Länder aus eigener Kraft erbringen und welche Leistungen durch Sonderförderprogramme (quer-) finanziert werden.

\section{Literatur}

Augurzky B, Krolop S, Mensen A, Pilny A, Schmidt CM, Wuckel C (2018) Krankenhaus Rating Report 2018: Personal Krankenhäuser zwischen Wunsch und Wirklichkeit. medhochzwei, Heidelberg

DKG - Deutsche Krankenhausgesellschaft (Hrsg) (2007) Bestandsaufnahme zur Krankenhausplanung und Investitionsfinanzierung in den Bundesländern. Stand April 2007. Berlin

DKG - Deutsche Krankenhausgesellschaft (Hrsg) (2009) Bestandsaufnahme zur Krankenhausplanung und Investitionsfinanzierung in den Bundesländern. Stand Juli 2009. Berlin

DKG - Deutsche Krankenhausgesellschaft (Hrsg) (2018) Bestandsaufnahme zur Krankenhausplanung und Investitionsfinanzierung in den Bundesländern. Stand Juni 2018. Berlin

RWI (2017) Stand und Weiterentwicklung der Investitionsförderung im Krankenhausbereich. RWI Projektberichte

Statistisches Bundesamt (Hrsg) (2011) Grunddaten der Krankenhäuser 2010: Fachserie 12: Gesundheitswesen, Reihe 6.1.1. Wiesbaden

Statistisches Bundesamt (Hrsg) (2013a) Grunddaten der Krankenhäuser 2011: Fachserie 12: Gesundheitswesen, Reihe 6.1.1. Wiesbaden

Statistisches Bundesamt (Hrsg) (2013b) Grunddaten der Krankenhäuser 2012: Fachserie 12: Gesundheitswesen, Reihe 6.1.1. Wiesbaden 
Statistisches Bundesamt (Hrsg) (2014) Grunddaten der Krankenhäuser 2013: Fachserie 12: Gesundheitswesen, Reihe 6.1.1. Wiesbaden

Statistisches Bundesamt (Hrsg) (2015) Grunddaten der Krankenhäuser 2014: Fachserie 12: Gesundheitswesen, Reihe 6.1.1. Wiesbaden

Statistisches Bundesamt (Hrsg) (2016) Grunddaten der Krankenhäuser 2015: Fachserie 12: Gesundheitswesen, Reihe 6.1.1. Wiesbaden
Statistisches Bundesamt (Hrsg) (2017) Grunddaten der Krankenhäuser 2016: Fachserie 12: Gesundheitswesen, Reihe 6.1.2. Wiesbaden

Statistisches Bundesamt (Hrsg) (2018a) Preise - Preise und Preisindizes für gewerbliche Produkte (Erzeugerpreise). Juni 2018: Fachserie 17, Reihe 2. Wiesbaden

Statistisches Bundesamt (Hrsg) (2018b) Volkswirtschaftliche Gesamtrechnungen. Inlandsproduktsberechnung. Lange Reihen ab 1970. 2017: Fachserie 18, Reihe 1.5. Wiesbaden

Open Access Dieses Kapitel wird unter der Creative Commons Namensnennung 4.0 International Lizenz(http://creativecommons. org/licenses/by/4.0/deed.de) veröffentlicht, welche die Nutzung, Vervielfältigung, Bearbeitung, Verbreitung und Wiedergabe in jeglichem Medium und Format erlaubt, sofern Sie den/die ursprünglichen Autor(en) und die Quelle ordnungsgemäß nennen, einen Link zur Creative Commons Lizenz beifügen und angeben, ob Änderungen vorgenommen wurden.

Die in diesem Kapitel enthaltenen Bilder und sonstiges Drittmaterial unterliegen ebenfalls der genannten Creative Commons Lizenz, sofern sich aus der Abbildungslegende nichts anderes ergibt. Sofern das betreffende Material nicht unter der genannten Creative Commons Lizenz steht und die betreffende Handlung nicht nach gesetzlichen Vorschriften erlaubt ist, ist für die oben aufgeführten Weiterverwendungen des Materials die Einwilligung des jeweiligen Rechteinhabers einzuholen. 